\title{
THE DIAMETERS OF THE GRAPHS OF SEMIRINGS
}

\author{
J. S. RATTI and Y.-F. LIN
}

(Received 23 June 1969)

Communicated by G. B. Preston

\section{Introduction}

Let $\mathscr{F}$ be a family of sets, $\left\{F_{\alpha} \mid \alpha \in A\right\}$. By the graph $G(\mathscr{F})$ of the system $\mathscr{F}$, we mean the graph whose set of vertices is $\mathscr{F}$ and in which the vertices $F_{\alpha}$, $F_{\beta} \in \mathscr{F}$ are adjacent (that is, are joined by an edge) if and only if $F_{\alpha} \neq F_{\beta}$ and $F_{\alpha} \cap F_{\beta} \neq \square$, where $\square$ denotes the empty set.

Definttion 1 . Let $\mathscr{F}$ be a family of sets, a subfamily $\left\{F_{1}, F_{2} \cdots, F_{n}\right\}$ of $\mathscr{F}$ forms a path, or a chain, between $F_{1}$ and $F_{n}$ in the graph $G(\mathscr{F})$ if and only if $F_{i} \cap F_{i+1} \neq \square$ for all $i=1, \cdots, n-1$. A graph is said to be connected provided, for every pair of vertices there is a path between them.

Definition 2. The distance $d\left(F_{\alpha}, F_{\beta}\right)$ between two vertices $F_{\alpha}$ and $F_{\beta}$ of a graph is the number of edges in a shortest path between these vertices (if no such path exists, we define $d\left(F_{\alpha}, F_{\beta}\right)=+\infty$; of course $d\left(F_{\alpha}, F_{\alpha}\right)=0$ ). The diameter of a graph is the supremum of $d\left(F_{\alpha}, F_{\beta}\right)$, where $\left(F_{\alpha}, F_{\beta}\right)$ runs over all pairs of vertices of the graph.

Definition 3. A semiring is a non-empty set $R$ equipped with two binary operations, called addition + and multiplication (denoted by juxtaposition), such that $R$ is multiplicatively a semigroup, additively a commutative semigroup and multiplication is distributive across the addition.

We have the following well-known theorem.

THEOREM A [1]. For any graph $G$ there exists a system $\mathscr{F}$ of sets such that the graph $G$ is isomorphic with the graph $G(\mathscr{F})$.

Theorem A shows that the general case is not very interesting. It would be of interest to know more information about the graph $G(\mathscr{F})$, when the members of $\mathscr{F}$ have an algebraic structure. The first step in this direction was taken by Bosák [1].

Throughout this paper, let $S$ be a given semigroup and $\mathscr{S}$ be the system of all proper subsemigroups of $S$; let $R$ be a given semiring and $\mathscr{R}$ the family of all proper subsemirings of $R$. 
Bosák [1] proved the following theorem.

THEOREM B. Let $S$ be a periodic semigroup with more than two elements. Then its graph $G(\mathscr{S})$ is connected and the diameter $D(S)$ of this graph is equal to:

(i) 0 if $S$ is a cyclic group of prime order;

(ii) 1 if $S$ has a single idempotent, but $S$ is not a cyclic group of prime order;

(iii) 3 if there exist in $S$ two idempotents $u \neq v$ such that $S=\langle u, v\rangle$ (that is, $S$ is the semigroup generated by the idempotents $u, v$ as its generators);

(iv) 2 in the remaining cases.

Bosák then raised the following open problem: Does there exist a semigroup with more than two elements whose graph is disconnected?

Lin [2] answered Bosák's problem by proving the following theorem:

THEOREM C. The graph of every semigroup with more than two elements is connected.

In [3] we discussed the graph $G(\mathscr{R})$ of a semiring $R$ and posed the following

CONJECTURE. The graph of every semiring with more than two elements is connected.

Although we could not prove our conjecture for an arbitrary semiring $R$, we did prove it for the cases (i) $R$ is left unital (ii) $R$ is normal (iii) $R$ is commutative (iv) $R$ is uncountable.

In $\S 2$ we prove that for some special semirings $R$ the diameter $D(R)$ of the graphs $G(\mathscr{R})$ is $\leqq 3$.

\section{The diameter of the graph of a semiring}

In this section we discuss the diameter of the graphs of some special types of semirings.

THEOREM 1. The diameter of the graph of a left unital semiring with more than two elements does not exceed three.

Proof. Let $R$ be such a semiring with left unit $e$. Let $R_{1}$ and $R_{2}$ be any two disjoint proper subsemirings of $R$, and let $a \in R_{1}$ and $b \in R_{2}$ be two arbitrary fixed elements. We shall construct a path, in $G(\mathscr{R})$, of length at most three between $R_{1}$ and $R_{2}$. Clearly, either $\left\{R_{1},\langle a, 2 e\rangle,\langle 2 e, b\rangle, R_{2}\right\}$ is a path, or else $\langle a, 2 e\rangle=R$, or $\langle 2 e, b\rangle=R$ (throughout this paper, $\left\langle x_{1}, \cdots, x_{n}\right\rangle$ means the subsemiring generated by $x_{1}, \cdots x_{n}$, as its generators). Let us assume $\langle a, 2 e\rangle=R$; the case $\langle 2 e, b\rangle=R$ can be handled similarly.

It is sufficient to construct a proper subsemiring $R_{\alpha}$ of $R$ such that $R_{1} \cap R_{\alpha} \neq \square$ and $R_{\alpha} \cap\langle e\rangle \neq \square$. Since if this has been established, then similarly there must exist $R_{\beta}$ in $G(\mathscr{R})$ such that $R_{\beta} \cap\langle e\rangle \neq \square$ and $R_{\beta} \cap R_{2} \neq \square$. Consequently, 
$\left\{R_{1}, R_{\alpha}, R_{\beta}, R_{2}\right\}$ will be a path of length 3 . To this end, we divide the rest of the proof into the cases (1) $2 e=e$ and $a^{2}=a$, (2) $2 e=e$ and $a^{2} \neq a$, and (3) $2 e \neq e$.

CASE 1: $2 e=e$ and $a^{2}=a$.

(1.1) $a=e$. In this case we choose $R_{\alpha}=\langle a\rangle=\langle e\rangle$

(1.2) $a \neq e$. In this case we have semiring $R=\{e, a, a e, e+a, e+a e, a+a e$, $e+a+a e\}$, with the following multiplication table (the addition table, which may be constructed easily is omitted for the sake of space saving).

\begin{tabular}{llllllll}
\hline & $e$ & $a$ & $a e$ & $e+a$ & $e+a e$ & $a+a e$ & $e+a+a e$ \\
\hline & $e$ & $a$ & $a e$ & $e+a$ & $e+a e$ & $a+a e$ & $e+a+a e$ \\
$a e$ & $a e$ & $a$ & $a e$ & $a+a e$ & $a e$ & $a+a e$ & $a+a e$ \\
$e+a$ & $a e$ & $a$ & $a e$ & $a+a e$ & $a e$ & $a+a e$ & $a+a e$ \\
$e+a e$ & $e+a e$ & $a$ & $a e$ & $e+a+a e$ & $e+a e$ & $a+a e$ & $e+a+a e$ \\
$a+a e$ & $e+a e$ & $a$ & $a e$ & $e+a+a e$ & $e+a e$ & $a+a e$ & $e+a+a e$ \\
$e+a+a e$ & $a e$ & $a$ & $a e$ & $a+a e$ & $a e$ & $a+a e$ & $a+a e$ \\
& $e+a e$ & $a$ & $a e$ & $e+a+a e$ & $e+a e$ & $a+a e$ & $e+a+a e$ \\
\hline
\end{tabular}

From the above multiplication table, we find the following two 'master' proper subsemirings:

and

$$
\{a, a e, e+a e, a+a e, e+a, e+a+a e\}
$$

$$
\{e, a e, e+a e, a+a e, e+a, e+a+a e\}
$$

Since the union of these two proper subsemirings contains $R$, the graph $G(\mathscr{R})$ is connected and $D(R)=3$.

CASE 2. $2 e=e$ and $a^{2} \neq a$. In this case we chose $R_{\alpha}=\left\langle e, a^{3}\right\rangle$, unless $R_{\alpha}=R$. Assume $R=\left\langle e, a^{3}\right\rangle$. Since $a \in R$, we have the following possibilities:

(2.1) $a=e$. This cannot happen, since $a^{2} \neq a$.

(2.2) $a=a^{3 l_{1}}+a^{3 l_{2}}+\cdots+a^{3 l_{n}}$, where $l_{i}, i=1, \cdots, n$, are positive integers.

Let $p(a)=a^{3 l_{1}-1}+\cdots+a^{3 l_{n}-1}$. Then (2.2) gives $a=a p(a)$; which implies $p^{2}(a)=p(a)$ and the proof follows from Case 1 by replacing $a$ by $p(a)$.

(2.3) $a=e+q\left(a^{3}\right)+r\left(a^{3}\right) e$, for some $q\left(a^{3}\right)$ and $r\left(a^{3}\right)$ in $\left\langle a^{3}\right\rangle$. Since $a \in R_{1} \cap(e+R)$ and $e=2 e \in(e+R) \cap\langle e\rangle$; we may choose $R_{\alpha}=e+R$, unless $e+R=R$. Assume $e+R=R$. For $x \in R$, there exists $y \in R$ such that $x=e+y$; and $e+x=e+(e+y)=2 e+y=e+y=x$. Thus, $e$ functions as the additive zero for $R$. Hence from (2.3) we get $a=q\left(a^{3}\right)+r\left(a^{3}\right) e$. By multiplying this last expression by $a$, we get $a^{2}=a^{2} Q(a)$ where $Q(a)=a^{3 l_{1}-1}+\cdots+a^{3 l_{n}-1}$, for some integers $l_{1}, \cdots, l_{n}$.

This implies that $Q^{2}(a)=Q(a)$. Thus the proof follows from Case 1 by replacing $a$ by $Q(a)$. 
(2.4) $a=e+q\left(a^{3}\right)$.

(2.5) $a=q\left(a^{3}\right)+r\left(a^{3}\right) e$.

(2.6) $a=r\left(a^{3}\right) e$

(2.7) $a=e+r\left(a^{3}\right) e$.

The subcases (2.4), (2.5), (2.6), and (2.7) are similar to the subcase (2.3), the proof for these cases is therefore omitted.

CASE 3. $e+e \neq e$. In this case we have $R=\langle 2 e, a\rangle$, because otherwise we choose $R_{\alpha}=\langle 2 e, a\rangle$. Since $e \in R, e$ can be expressed as:

(3.1) $e=2 m e$ for some integer $m>1$.

Let $e_{1}=(2 m-1) e$, then $e_{1}+e_{1}=e_{1}$ and $e_{1}^{2}=e_{1}$. Since $(2 m-1) a=e_{1} a \in$ $R_{1} \cap e_{1} R$ and $(2 m-1) e \in e_{1} R \cap\langle e\rangle$. We must have $R=e_{1} R$ (if $R \neq e_{1} R$, we choose $R_{\alpha}=e_{1} R$ ). Since $e_{1}^{2}=e_{1}$ and $e_{1} R=R$, it is easily seen that $e_{1}$ is a left unit for $R$ with $e_{1}+e_{1}=e_{1}$, and the proof follows from cases 1 and 2 .

(3.2) $e=f(a)$ for some $f(a) \in\langle a\rangle$.

Since $e=f(a)$, we have $R=\langle 2 e, a\rangle \subset R_{1} \neq R, a$ contradiction.

(3.3) $e=p(a) e$ for some $p(a) \in\langle a\rangle$.

Since $p(a) \in R_{1} \cap\langle 2 e, p(a)\rangle$ and $2 e \in\langle 2 e, p(a)\rangle \cap\langle e\rangle$, we have $R=\langle 2 e$, $p(a)\rangle$ (otherwise choose $R_{a}=\langle 2 e, p(a)\rangle$ ). Also the equation $e=p(a) e$ gives $a=p(a) a$, which implies that $p^{2}(a)=p(a)$. Since $e \in R=\langle 2 e, p(a)\rangle$, we have the following possibilities:

(3.3.1) $e=2 m e$. This case is the same as subcase (3.1) already discussed.

(3.3.2) $e=n p(a)$ for some integer $n \geqq 1$. This case is similar to the subcase (3.2).

(3.3.3) $e=2 n p(a) e=2 n e$. This is the subcase (3.1).

(3.3.4) $e=n p(a)+2 m p(a) e$. This equation gives $e=e^{2}=n p(a) e+2 m p(a) e$ $=(n+2 m) e$, which is the subcase (3.1).

(3.3.5) $e=2 m e+n p(a)$. We again have $e=e^{2}=2 m e+n p(a) e=(2 m+n) e$, which is the subcase (3.1).

(3.3.6) $e=2 n e+m p(a) e$.

(3.3.7) $e=2 n e+m p(a)+2 l p(a) e$.

The cases (3.3.6) and (3.3.7) can be similarly handled.

(3.4) $e=f(a)+p(a) e$, for some $f(a)$ and $p(a)$ in $\langle a\rangle$. In this case we have $e=e^{2}=f(a) e+p(a) e=[f(a)+p(a)] e=h(a) e$, where $h(a)=f(a)+p(a) \in\langle a\rangle$. The subcase (3.3) now applies.

(3.5) $e=2 m e+f(a) e$ for some integer $m \geqq 1$ and $f(a) \in\langle a\rangle$. 
Let $d=(2 m-1) e+f(a) e=d_{1} e$ where $d_{1}=(2 m-1) e+f(a)$. With this notation, we get $e=e+d$, and hence

$$
\begin{aligned}
d+d & =(2 m-1) e+f(a) e+d \\
& =[(2 m-1) e+d]+f(a) e \\
& =(2 m-1) e+f(a) e \\
& =d
\end{aligned}
$$

By squaring both sides of the equality $e=e+d$, we obtain $e=e+d^{2}$. Consequently,

$$
d+d^{2}=[f(a) e+(2 n-1) e]+d^{2}=f(a) e+(2 n-1) e=d,
$$

while

$$
\begin{aligned}
d+d^{2} & =d+[(2 m-1) e+f(a) e]^{2} \\
& =d+(2 m-1)^{2} e+2(2 m-1) f(a) e+f^{2}(a) e \\
& =\left[d+(2 m-1)^{2} e\right]+2(2 m-1) f(a) e+f^{2}(a) e \\
& =(2 m-1)^{2} e+2(2 m-1) f(a) e+f^{2}(a) e \\
& =d^{2}
\end{aligned}
$$

Thus $d=d^{2}$.

Let $R_{d_{1}}=\left\{x \mid x \in R\right.$ and $\left(x+d_{1}\right) e=2 k e$ for some positive integer $\left.k\right\}$. If $x_{1}, x_{2} \in R_{d_{1}}$, then $x_{1}+d_{1}=2 k_{1} e$ and $x_{2}+d_{1}=2 k_{2} e$. Consequently

$$
\begin{aligned}
\left(x_{1}+x_{2}+d_{1}\right) e & =\left(x_{1}+x_{2}\right) e+d \\
& =\left(x_{1}+x_{2}\right) e+2 d=\left(x_{1}+d_{1}\right) e+\left(x_{2}+d_{1}\right) e \\
& =2\left(k_{1}+k_{2}\right) e,
\end{aligned}
$$

and

$$
\begin{aligned}
4 k_{1} k_{2} e & =\left[\left(x_{1}+d_{1}\right) e\right]\left[\left(x_{2}+d_{1}\right) e\right] \\
& =\left(x_{1} e+d\right)\left(x_{2} e+d\right) \\
& =x_{1} x_{2} e+x_{1} d+d x_{2} e+d^{2} \\
& =x_{1} x_{2} e+x_{1} d+d^{2}+d x_{2} e+d^{2}, \quad \text { since } d^{2}=2 d^{2} \\
& =x_{1} x_{2} e+\left(x_{1}+d\right) d+d x_{2} e+d^{2} e, \quad \text { since } d^{2} e=d^{2} \\
& =x_{1} x_{2} e+\left(x_{1}+d\right) e d+d\left(x_{2}+d\right) e \\
& =x_{1} x_{2} e+2 k_{1} e d+d\left(2 k_{2} e\right) \\
& =x_{1} x_{2} e+2 k_{1} d+2 k_{2} d \\
& =x_{1} x_{2} e+d \\
& =\left(x_{1} x_{2}+d_{1}\right) e .
\end{aligned}
$$


Since $2 e \in R_{d_{1}}, R_{d_{1}} \neq \square$. Consequently $R_{d_{1}}$ is a subsemiring of $R$. Also

$$
\begin{aligned}
{\left[d_{1}+f^{2}(a)\right] e } & =d+f^{2}(a) e \\
& =(2 m-1)[(2 m-1) e+f(a) e]+f^{2}(a) e \\
& =(2 m-1)^{2} e+f(a)[(2 m-1) e+f(a)] e \\
& =(2 m-1)^{2} e+f(a) d \\
& =(2 m-1)^{2} e+(2 m-1) d+f(a) d, \quad \text { since } e+d=e \\
& =(2 m-1)^{2} e+[(2 m-1) e+f(a) e] d, \quad \text { since } \quad e d=d \\
& =(2 m-1)^{2} e+d^{2} \\
& =(2 m-1)^{2} e .
\end{aligned}
$$

Thus,

$$
\begin{aligned}
\left(2 f^{2}(a)+d_{1}\right) e & =2 f^{2}(a) e+d \\
& =2 f^{2}(a) e+2 d \\
& =2\left(f^{2}(a) e+d\right) \\
& =2(2 m-1)^{2} e .
\end{aligned}
$$

Therefore, $2 f^{2}(a) \in R_{d_{1}}$.

Now $2 f^{2}(a) \in R_{1} \cap R_{d_{1}}$ and $2 e \in R_{d_{1}} \cap\langle e\rangle$, we have $R_{d_{1}}=R$ (otherwise choose $R_{a}=R_{d_{1}}$ ). fore,

Since $e \in R=R_{d_{1}}$, we have $\left(e+d_{1}\right) e=2 k e$ for some positive integer $k$. There-

$$
2 k e=\left(e+d_{1}\right) e=e+d_{1} e=e+d=e
$$

and the proof follows from subcase (3.1).

(3.6) $e=2 m e+f(a)$ for some positive integer $m$ and $f(a) \in\langle a\rangle$. In this case $e=e^{2}=2 m e+f(a) e$, and which reduces to (3.5).

(3.7) $e=2 m e+f(a)+2 n p(a) e$. We again have

$$
\begin{aligned}
e & =e^{2}=2 m e+(f(a)+2 n p(a)) e \\
& =2 m e+h(a) e, \text { where } h(a) \in\langle a\rangle
\end{aligned}
$$

and this case also reduces to (3.5).

THEOREM 2. The diameter of the graph of a commutative semiring $R$ with more than elements does not exceed three.

Proof. Let $R_{1}$ and $R_{2}$ be two disjoint proper subsemirings of $R$, and let $a \in R_{1}$ and $b \in R_{2}$ be any two fixed elements. Then $\left\{R_{1}, a R, R b, R_{2}\right\}$ is a path of length three between $R_{1}$ and $R_{2}$, unless $a R=R$ or $R b=R$. Assume $a R=R$ (the case $R b=R$ may be handled similarly). Since $R$ is commutative, we have $R=a R=R a$. It follows that there exists an element $e \in R$ such that $a=e a$. For each $x \in R, R=a R$ implies that there exists an element $y \in R$ such that $x=a y$. 
Thus,

$$
e x=e(a y)=(e a) y=a y=x,
$$

which shows that $R$ is left unital and thus, by Theorem 1 , the diameter of the graph $G(\mathscr{R})$ is $\leqq 3$.

THEOREM 3. The graph of a semiring $R$ with more than two elements, having ascending chain condition (A.C.C.) or descending chain condition (D.C.C.), is connected and the diameter $D(R)$ does not exceed three.

Proof. Let $R_{1}$ and $R_{2}$ be two disjoint proper subsemirings of $R$ and let $a \in R_{1}$ and $b \in R_{2}$ be two fixed elements. We observe that $\left\{R_{1}, a R, R b, R_{2}\right\}$ is a path of length at most 3 between $R_{1}$ and $R_{2}$ unless $a R=R$ or $R b=R$. Assume $a R=R$ (the case $R b=R$ may be similarly handled). Since $2 a=a+a \in R_{1} \cap(R+R)$ and $2 b=b+b \in R_{2} \cap(R+R),\left\{R_{1}, R+R, R_{2}\right\}$ is a path between $R_{1}$ and $R_{2}$ unless $R+R=R$. Let us assume that $R+R=R$.

Let

$$
A=\{x \mid x \in R \text { and } x R=R\} .
$$

It is easily seen that $A$ is a subsemiring of $R$ and $a \in A$.

Suppose $b \notin A$, i.e. $b R \in \mathscr{R}$. In this case $\left\{R_{1}, R a, b R, R_{2}\right\}$ is a path between $R_{1}$ and $R_{2}$, unless $R a=R$. Assume $R a=R$. We then have

$$
R a=R=a R,
$$

which implies that $R$ is left unital and the proof follows from Theorem 2 .

On the other hand if $b \in A$, then $\left\{R_{1}, A, R_{2}\right\}$ is a path between $R_{1}$ and $R_{2}$, unless $A=R$. Let us assume that $A=R$.

CASE 1. $R$ satisfies A.C.C.

Since $a R=R$, there exists a sequence $\left\{x_{i}\right\} \subset R$ such that $a=a x_{1}=a^{2} x_{2}=\cdots$, where $x_{i}=a x_{i+1}$ for $i=1,2, \cdots$. We then have $R a \subset R x_{1} \subset R x_{2} \subset \cdots$. Since $R$ satisfies A.C.C. there exists an $n$ such that $R x_{n}=R x_{n+1}$, which implies that $x_{n}=a x_{n+1} \in R x_{n+1}=R x_{n}$. Thus there exists an element $e \in R$ such that $x_{n}=e x_{n}$. We also have $x_{n} R=R$ (since $A=R$ ). Let $x \in R$. There exists $y \in R$ such that $x=x_{n} y$ and $e x=e\left(x_{n} y\right)=\left(e x_{n}\right) y=x_{n} y=x$. Thus $e$ is a left unit for $R$ and the proof follows from Theorem 1 .

CASE 2. $R$ satisfies D.C.C.

It $R$ satisfies D.C.C., we see from $R a \supset R a^{2} \supset R a^{3} \supset \cdots$, that for some $m$; $R a^{m}=R a^{m+1}$. Thus $a^{m+1} \in R a^{m}=R a^{m+1}$, which implies that there exists an $e^{*}$ such that $a^{m+1}=e^{*} a^{m+1}$ and by the same argument as in Case 1 we can show that $e^{*}$ is a left unit for $R$ and the proof follows from Theorem 1 .

REMARK. In [3] we showed that the graph of a semiring $R$ with two elements is not necessarily connected. 
The following example shows that the diameter $D(R)$ of the graph of semiring $R$ is equal to three.

EXAMPLE. Let $R=\{e, a, b\}$ be a semiring with the following addition and multiplication tables. The graph $G(\mathscr{R})$ is illustrated in Fig. 1.

\begin{tabular}{|c|c|c|c|c|c|c|c|}
\hline+ & $e$ & $a$ & $b$ & - & $e$ & $a$ & $b$ \\
\hline$e$ & $e$ & $b$ & $b$ & $e$ & $e$ & $a$ & $b$ \\
\hline$a$ & $b$ & $a$ & $b$ & $a$ & $a$ & $a$ & $a$ \\
\hline$b$ & $b$ & $b$ & $b$ & $b$ & $b$ & $a$ & $b$ \\
\hline
\end{tabular}

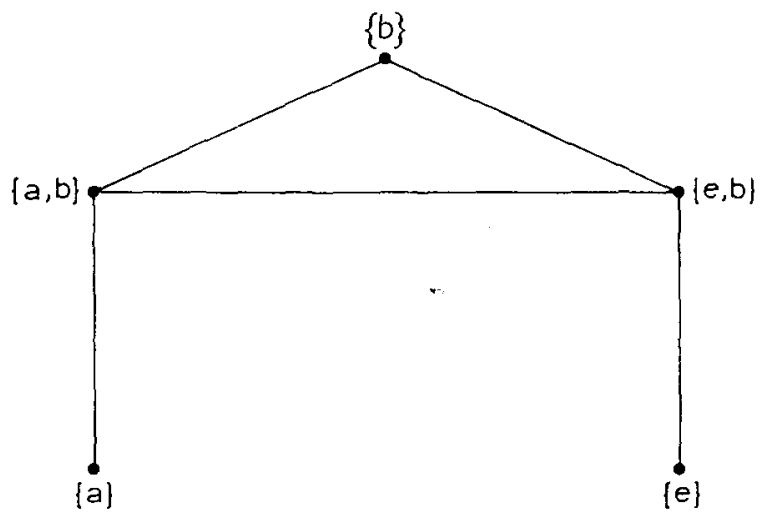

Figure 1

\section{References}

[1] J. Bosák, The graphs of semigroups, theory of graphs, and its applications Proc. Symp. Graph Theory, Smolenice 1(963), Academic Press (1965), pp. 119-125.

[2] Y-F. Lin, 'A problem of Bosák concerning the graphs of semigroups', Proc. American Math. Society 21 (1969), 343-346.

[3] Y.-F. Lin and J. S. Ratti, 'The graphs of semirings', Journal of Algebra 14 (1970), 73-82.

University of South Florida

Tampa, Florida 